\title{
Cáncer oral. Métodos de diagnóstico (screening) rápido en la consulta odontológica
}

\author{
Barbany JR*
}

\section{RESUMEN}

Se analizan los procedimientos existentes en el mercado, destinados al diagnóstico rápido de lesiones de la mucosa oral sospechosas de malignidad, en la consulta odontológica. De entre los distintos métodos propuestos: tinción con colorantes, iluminación con luz fluorescente y biopsia optimizada con toma de muestras ("brush biopsy"), monitorizada por computadora y valorada por un equipo específico de anátomo-patólogos, éste último es el que más documentado y con un mayor nivel de garantía y seguridad..

Palabras clave: Diagnóstico rápido cáncer oral en consulta odontológica, métodos de tinción, métodos de fluorescencia, biopsia con cepillo especial.

\section{SUMMARY}

Different proceedings marketed for a quick diagnostic of oral mucosa lesions, suspicious of cancer at the dentistry cabinet, are reviewed. Among the different methods proposed: colour staining, fluorescence light and optimized brush biopsy computer monitored and analyzed by a specially trained anatomopathological team, this one seems the most referenced with a better level of safety and confidence.

Key words: Quick screening oral cancer in dentistry cabinet, staining methods, fluorescence methods, brush biopsy.

Fecha de recepción: Enero 2008.

Aceptado para publicación: Febrero 2008.

* Departamento de Ciencias Fisiológicas II. Campus de Bellvitge. Universidad de Barcelona.

Barbany JR. Cáncer oral. Métodos de diagnóstico (screening) rápido en la consulta odontológica. Av. Odontoestomatol 2008; 24 (1): 123-128.

\section{INTRODUCCIÓN}

El cáncer oral es uno de los más frecuentes y con mayor índice de mortalidad, por lo que es interesante disponer de procedimientos que permitan efectuar un diagnóstico precoz, en particular en aquellas situaciones en las que el odontólogo se encuentra frente a una lesión de la mucosa oral sospechosa de riesgo, pero en la que la práctica de una biopsia, no se encuentra del todo justificada. Además de contribuir a completar el estudio clínico del paciente, estas herramientas de diagnóstico auxiliar, que no pueden excluir la obligada observación y seguimiento clínicos, son un elemento importante cara a garantizar 
un mayor grado de seguridad y de confianza para el paciente y el odontólogo y ayudan a evitar además, posibles reclamaciones que hipotéticamente el odontólogo debería afrontar, por no haber intervenido de forma eficaz, desde el punto de vista diagnóstico y preventivo frente a una lesión de naturaleza "precancerosa", en la boca de su paciente.

Con esta finalidad, han aparecido recientemente estrategias diagnósticas de distinta índole que tratan de complementar los datos proporcionados por la clínica, valorando los niveles de riesgo y aconsejando en caso de sospecha de malignidad, la práctica de una biopsia convencional sobre la lesión. Este tipo de procedimientos, son en esencia, comparables a otros introducidos hace ya tiempo en el ámbito de la prevención e incluidos en los protocolos de actuación, como pueden ser el frotis vaginal, con tinción de Papanicolaou, para el diagnóstico precoz del carcinoma de cuello de útero, las mamografías, las colonoscopias de rutina para el diagnóstico precoz del cáncer de intestino grueso o la valoración de los niveles séricos de antígeno prostático (PSA), como indicativo del riesgo de carcinoma de próstata.

El conjunto de métodos de diagnóstico precoz descritos, algunos de ellos con un uso comercial, pueden agruparse en:

\section{MÉtodos DE TINCIÓN CON COLORANTES}

Evidencian las diferencias en los niveles de actividad metabólica existentes entre las células normales y tumorales, lo que se traduce en una mayor afinidad por determinados colorantes.

\section{Azul de toluidina}

Son técnicas que llevan ya un cierto tiempo comercializadas, aunque se hallan en general cuestionadas y poco introducidas y con una utilización limitada. La que goza de mayor difusión, conocida con el nombre comercial de OraScan, fue introducida hace ya algunos años y consta de tres elementos: solución 1 ("pre rinse"); solución 2, en la que se contiene el colorante de toluidina, y solución 3 ("post rinse"). Una vez detectada en la mucosa oral la lesión que se presume como de riesgo, se aclara la cavidad bucal con aproximadamente la mitad de la solución 1: "pre rinse" (20 segundos) y expectoración; después se efectúa un aclarado con agua (20 segundos) y expectoración; a continuación lavado y difusión por la boca con la mitad de la solución 2 de toluidina durante 60 segundos y expectoración y después un nuevo aclarado ahora con la solución 3: "post rinse" durante 20 segundos y expectoración. Se repite la tinción utilizando para ello el remanente de la solución de toluidina y se procede a un nuevo aclarado con agua por dos veces. Se observa directamente la zona sospechosa, utilizando en caso necesario, una fuente luminosa adecuada y si es preciso, una lupa u otro procedimiento de magnificación. Las posibles lesiones y zonas de riesgo, se colorean en azul de manera que, una retención de colorante que no es eliminada por el aclarado, debe considerarse sospechosa, sobre la que deberá practicarse la biopsia convencional. Hay que tener en cuenta que algunas áreas específicas de la boca aunque pueden retener colorante, no se consideran como de riesgo, en concreto las que se corresponden con las papilas del dorso de la lengua y los márgenes gingivales alrededor del diente. En relación con este método la bibliografía es relativamente extensa (1-5), con resultados dispares, aunque en general predomina, un bajo nivel de especificidad.

Un procedimiento semejante es el del producto OraTest. De introducción más reciente, dispone de un menor bagaje clínico. En un trabajo efectuado sobre sesenta pacientes con de lesiones en la mucosa oral sospechosas de malignidad, el uso de OraTest, proporciona un índice de sensibilidad al teñido, de un 93,9\% en relación al carcinoma oral y del 42 '9\% respecto a las displasias. A partir de estos datos, puede concluirse que la utilidad de este producto como indicativo de riesgo de cáncer oral, no queda suficientemente demostrada $(6,7)$.

\section{Otros colorantes}

Con un fundamento y criterio semejante, otros investigadores han propuesto recientemente el uso de otros colorantes alternativos, en concreto azul de metileno, rosa de bengala y cloruro de tolonio. Se ha propuesto el uso de azul de metileno $(8,9)$, a partir de un trabajo de campo efectuado sobre un total de 
58 pacientes. Se obtiene una sensibilidad del $90 \%$ y una especificidad del $69 \%$. El valor de predicción positivo se estima cercano al $74 \%$ y el negativo equivale al $87 \%$. Los autores concluyen que aunque produce un considerable número de falsos positivos y falsos negativos, es más barato que el azul de toluidina, por lo que podría tener interés como elemento adicional de diagnóstico en zonas geográficas con pocos recursos y elevada incidencia de cáncer oral. La tinción debe completarse siempre con el correspondiente seguimiento clínico de la lesión y en caso necesario es imprescindible la confirmación de la malignidad mediante biopsia.

En otro estudio se ha utilizado como colorante rosa de bengala. Llevado a cabo sobre 132 pacientes, que presentaban displasia epitelial y riesgo de carcinoma escamoso (10), los autores proponen como novedad, valorar la intensidad de coloración alcanzada en 4 grados. Con este procedimiento, hallan valores de sensibilidad del 93,9\%, con una especificidad correspondiente al 73,7\%. La relación predictiva positiva es de 3,570 y la negativa de 0,082 , por lo que concluyen que puede ser un buen elemento de diagnóstico precoz de carcinoma escamoso y otras lesiones malignas.

En un estudio multicéntrico efectuado sobre un total de 668 pacientes, se ha propuesto el uso de cloruro de tolonio (11). Se compara el procedimiento con un examen visual efectuado por un segundo investigador y si la lesión aparece como sospechosa se practica biopsia. Los valores de sensibilidad son del 96,7\% en relación a la retención del colorante cloruro de tolonio y los valores predictivos resultantes son semejantes para el examen clínico y un test positivo para el colorante $(36,4 \%$ vs $32,6 \%)$, indicando que la mayor sensibilidad del tolonio no se asocia con más falsos positivos. De los resultados de su estudio los autores concluyen que el uso de este procedimiento de tinción, proporciona un nivel de sensibilidad más elevado que el simple examen clínico de inspección visual.

\section{MÉtodos basados en la ILUMinación CON LUZ FLUORESCENTE Y MÉTODOS COMBINADOS}

Como que la luz fluorescente es absorbida de forma distinta por el tejido epitelial sano o el anormal, se trata de someter la zona sospechosa a la iluminación directa mediante un foco de luz fluorescente y establecer el nivel de reflexión. En el tejido sano la absorción es completa (no refleja, no se ilumina o queda "oscura"), mientras que el tejido anormal, refleja la luz en forma de mancha blanca o de otras tonalidades.

Existen variantes para este procedimiento como el de la marca comercial Velscope, en el que además de la luz fluorescente, se utiliza una serie de filtros ópticos, que facilitan diferenciar los tejidos sanos, con una actividad metabólica que podría ser considerada como "normal" y que aparece entonces con un color verde manzana brillante, de los supuestamente malignos, con una tonalidad y aspecto oscuro. La bibliografía disponible estima su eficacia como procedimiento de rutina de uso en la consulta odontológica. $(12,13,14)$.

El método Vizi Lite, que como el anterior, se encuentra introducido en algunas consultas odontológicas especialmente en USA, es también un método basado en la luz fluorescente. Según el procedimiento inicial, se procede al aclarado de la boca con solución de ácido acético al $1 \%$ durante 1 minuto y expectorar. A continuación se activa la cápsula de quimioluminiscencia y se prepara el sistema retractor (ViziLiteR). Después de romper el vial y agitar la cápsula para mezclar los contenidos, se observa el reflejo de la luz de fluorescencia resultante, en unas condiciones de luz ambiente escasa, a fin de facilitar la visión de las posibles zonas sospechosas.

Actualmente se ha introducido una mejora, que se conoce con la denominación de ViziLite plus conjugando Vizi Lite, con un método de coloración adyuvante con azul de toluidina metacromático (Tblue 630). Este método mixto, combina la fluorescencia con la tinción por colorante y se revela como más útil que el precedente en la observación de la lesión sospechosa. Después del uso de la luz de fluorescencia se observa bajo luz normal, la coloración alcanzada después de la tinción con el colorante. En el caso que se detecte tejido anormal se practica la correspondiente consulta anatomopatológica. La bibliografía en relación con los procedimientos Vizi Lite, es abundante, aunque en general los resultados referenciados, no son demasiado predictivos (15-19). 


\section{MÉTOdo DE BIOPSIA OPTIMIZADA, EFECTUADA EN} LA CONSULTA Y PROCESADA EN LABORATORIO CENTRAL ("BRUSH BIOPSY")

Representado por el método OralCDx, se trata de un procedimiento por el que se procede a la toma de la muestra en la zona de la lesión sospechosa observada en el paciente, mediante un cepillo de características especiales y diseñado especialmente para tal fin. ("brush biopsy"). El grado de hemorragia producido en la toma de la muestra es mínimo y el procedimiento es indoloro para el paciente, por lo que no requiere el uso de anestesia tópica o local, sin excluir la posibilidad de su uso si así se desea. La muestra obtenida es depositada en un portaobjetos y sellada mediante un fijador. El portaobjetos se introduce en un recipiente plástico y mediante un sobre postal protegido, la muestra es remitida al laboratorio central, donde después de la correspondiente tinción, es analizada mediante un procedimiento automatizado en el que intervienen un sistema computadorizado de análisis de la imagen microscópica y un equipo especializado de patólogos que valoran la posible presencia de células anormales. El sistema computadorizado no efectúa el diagnóstico, reservado a un equipo de anatomopatólogos. A continuación se emite un informe detallado al dentista por fax y correo electrónico. El resultado obtenido se califica como: "Negativo": no hay evidencia de actividad o lesión precancerosa o cancerosa en la muestra; "Atipia": contiene células anormales que podrían indicar una lesión precancerosa. Contactar con el laboratorio para una información adicional y "Positivo": se aprecian células displásicas, indicativas de lesión precancerosa o cancerosa. En el supuesto que la muestra sea "Incompleta", por no contener un suficiente número de células epiteliales, debe procederse a una nueva prueba en el paciente, con un nuevo test, sin cargo. La abundante bibliografía existente sobre este método, destaca en general el buen nivel de predictividad y especificidad del sistema (20-44).

\section{PRocedimientos DE FUtURO}

En un futuro y en concordancia con el desarrollo tecnológico, van a desarrollarse nuevos procedimientos de diagnóstico, basados en la mejora de las técnicas de imagen y representación, como la tomo- grafía óptica tridimensional y la tomografía óptica por doppler, la tecnología fotónica, con obtención de imágenes no invasivas de las células "in vitro". El uso de endoscopios y microscopios utilizando procedimientos tomográficos de coherencia óptica y microscopia confocal de reflectancia, junto a los agentes de contraste específico con nanopartículas de oro con elevada afinidad para biomarcadores celulares específicos, permitirán la obtención de imágenes tridimensionales, con un alto grado de resolución Bajo otro prisma, profundizar en el estudio genético de marcadores moleculares o la posibilidad de obtener y analizar muestras de RNA procedente de células obtenidas por "brush biopsy", analizando posibles mutaciones cancerígenas o cambios en los genes supresores de tumores representan otras líneas de investigación. Parámetros indicativos de alteraciones epigenéticas, modificaciones del genoma o detección de inestabilidad en determinadas zonas del mismo, podrían ser útiles también como biomarcadores. Finalmente las tecnologías de "lap on a chip" (LOC), que empiezan a ser desarrolladas para el diagnóstico de tumores de distinta localización, son otro elemento diagnóstico importante de futuro $(43,45,46)$

\section{BIBLIOGRAFÍA}

1. Warnakulasuriya KA y Johnson NW. "Sensitivity and specificity of OraScan (R) toluidine blue mouthrinse in the detection of oral cancer and precancer". J Oral Pathol Med. 1996;25(3):97-103.

2. Eslami M, Keyhani A, Motahhary P, Agha-Hosseini Sharifzadeh F. "Comparison and Evaluation of the Reliability of Oratest and Generic Toluidine Blue in the Detection of Oral Malignancy and Premalignancy". J. Dentistry, Tehran University of Medical Sciences 2005;Vol:2, No.3.

3. Ather Siddiqui, Umer Farooq M, Riaz Ahmed Siddiqui, Tariq Rafi SM. "Role of Toluidine Blue in Early Detection of Oral Cancer" Imtiaz Pak J Med Sci 2006;22:184-7.

4. Kövesi G. OraTest. A new method for differentiating between benign and malignant oral tumors. Fogorv Sz. 1998;91:107-16. 
5. Bobby K. J: "Oral Cancer: Prevention and Detection" Medical Principles and Practice 2002;11:32-5.

6. Cheng B, Yang L: "The clinical evaluation of Oratest in detecting oral mucosal lesions" 2003;20-21:120-4

7. Hua Xi Kou Qiang Yi Xue Za Zhi, Cheng B, Yang L. "The clinical evaluation of Oratest in detecting oral mucosal lesions" 2003;20-21(2):124-6.

8. Chen YW, Lin JS, Fong JH, Wang IK, Chou SJ, Wu $\mathrm{CH}$, Lui MT, Chang CS, Kao SY. "Use of methylene blue as a diagnostic aid in early detection of oral cancer and precancerous lesions". $\mathrm{Br} \mathrm{J}$ Oral Maxillofac Surg. 2007;45:590-1.

9. Chen YW, Lin JS, Wu CH, Lui MT, Kao SY, Fong Y. "Application of in vivo stain of methylene blue as a diagnostic aid in the early detection and screening of oral squamous cell carcinoma and precancer lesions”. J Chin Med Assoc. 2007;70: 497-503.

10. Du GF, Li CZ, Chen HZ, Chen XM, Xiao Q, Cao ZG, Shang SH, Cai X. "Rose bengal staining in detection of oral precancerous and malignant lesions with colorimetric evaluation: A pilot study" Early Detection 2007;120:1958-63.

11. Joel MHS, Stephen R. Porter, MD, Roy Feldman, Rowena J. Dolor, MD "The utility of tolonium chloride rinse in the diagnosis of recurrent or second primary cancers in patients with prior upper aerodigestive tract cance" 2003;25:911-21.

12. Kois JC, Truelove E. "Detecting oral cancer: a new technique and case reports". Dent Today 2006;25(94):96-7.

13. Poh Lewei C, Zhang D, Anderson J, Scott Durham P, Williams R, Priddy K, Berean S, Tseng O, Calum O, Rosi M P: "Fluorescence Visualization Detection of Field Alterations in Tumor Margins of Oral Cancer Patients". Clin Cancer Res 2006:12.

14. Balevi B. "Evidence-based decision making: should the general dentist adopt the use of the VELscope for routine screening for oral cancer?". J Can Dent Assoc. 2007;7:603-6.
15. Farah CS, McCullough MJ. "A pilot case control study on the efficacy of acetic acid wash and chemiluminescent illumination (ViziLite) in the visualisation of oral mucosal white lesions". Oral Oncol. 2007;43:820-4.

16. Epstein JB, Silverman S Jr, Epstein JD, Lonky SA, Bride MA. "Analysis of oral lesion biopsies identified and evaluated by visual examination, chemiluminescence and toluidine blue". Oral Oncol. 2007.

17. Oh ES, Laskin DM: "Efficacy of the ViziLite system in the identification of oral lesions". J Oral Maxillofac Surg. 2007;65:424-6.

18. Kerr AR, Sirois DA, Epstein JB. "Clinical evaluation of chemiluminescent lighting: an adjunct for oral mucosal examinations". J Clin Dent. 2006;17:59-63. Epstein JB, Gorsky M, Lonky S, Silverman S Jr, Epstein JD, Bride M.: "The efficacy of oral lumenoscopy (ViziLite) in visualizing oral mucosal lesions". Spec Care Dentist. 2006;26:171-4.

19. Ram S, Siar CH. "Chemiluminescence as a diagnostic aid in the detection of oral cancer and potentially malignant epithelial lesions". Int J Oral Maxillofac Surg. 2005;34:521-17.

20. Sciubba JJ and the U.S. Collaborative OralCDx Study Group. "Improving detection of precancerous and cancerous oral lesions: Computerassisted analysis of the oral brush biopsy". U.S. Collaborative OralCDx Study Group. J Am Dent Assoc 1999; 130:1445-57.

21. Scheifele C, Schmidt-Westhausen AM, Dietrich T, Reichart PA. "The sensitivity and specificity of the OralCDx technique: evaluation of 103 cases". Oral Oncol. 2004;40:824-8.

22. Svirsky JA, Burns JC, Carpenter WM, et al. "Comparison of computer-assisted brush biopsy results with follow up scalpel biopsy and histology". Gen Dent 2002;50:500.

23. Kosicki DM, Riva C, Pajarola GF, Burkhardt A, Gratz K. "OralCDx brush biopsy- A tool for early diagnosis of oral squamous cell carcinoma". Schweiz Monatsschr Zahnmed 2007;117:222-7. 
24. Christian DC. Computer-assisted analysis of oral brush biopsies at an oral cancer screening program". J Am Dent Assoc 2002;133: 357-62.

25. Radensky PW, Archer JW, "Cost-Effectiveness of Evaluating Benign-looking Lesions Detected During Routine Oral Examination”. En preparación.

26. Flaitz CM, Felefli S. The Oral Brush Biopsy: "It's as Easy as 1,2,3". Texas Dental Journal 2000;117: 20-4.

27. Zunt SL. "Transepithelial Brush Biopsy: an adjunctive diagnostic procedure". J Indiana Dent Assoc 2001;80(2):6-8.

28. Eisen D. "The oral brush biopsy: a new reason to screen every patient for oral cancer". Gen Dent 2000;48:96-9.

29. Svirsky JA, Burns JC, Page, DG, Abbey, LM. "Computer-assisted analysis of the oral brush biopsy”. Compendium 2001;22:99-106.

30. Tauberg JA An oral surgeon's view of the computer-assisted oral brush biopsy. Alpha Omegan 2002;95:9-11.

31. Drinnan, AJ. "Screening for Oral Cancer and Precancer- a valuable new technique". General Dentistry 2000;48:656-60.

32. Sciubba JJ. "Oral brush biopsy with computerassisted analysis". eMedicine San Francisco, A:eMedicine.com; http://www.emedicine.com/ derm/topic701.htm.

33. Glazer H. "Detection of precancers and cancers of the oral cavity with computer assisted analysis of the brush biopsy". Independent Dentistry (London, UK): 2002;7 (3):85-9.

34. Gurenlian JR. "The brush biopsy: A chairside technique for early detection of oral cancer". Access 2003; September/October:32-6.

35. Kerr AR, Cruz GD. "Oral cancer. Practical prevention and early detection for the dental team". N Y State Dent J 2002;68:44-54.
36. Laskin DM. "Early diagnosis of squamous carcinoma”. J Oral Maxillofacial Surg 2003;61:851-2.

37. Stahl S, Meskin LH, Brown LJ. "The American Dental Association's oral cancer campaign: the impact on consumers and dentists". J Am Dent Assoc 2004;13:1261-7.

38. Oral Cancer. "The American Association of Oral and Maxillofacial Surgeons (AAOMS) Surgical Update". 2003;18:1-7.

39. American Dental Association: "Dental Product Spotlight". Journal of the American Dental Association 2001;132: 215.

40. Glazer H. Oral Cancer: "Be sure or get sued". AGD Impact 2002;30:16.

Moore L. "Advancing technologies for oral cancer screening". J California Dental Hygienists' Association 2003;18:18-24.

41. Curry JT. "Benefits of the brush biopsy". J Oral Maxillofacial Surg 2003;61:1506.

42. Edwards J. "New test aids in early diagnosis". Access 2000;14:25.

Leonard, M. "New Techniques for Diagnosing Oral Cancer”. Dental Economics (2000); June: 126-31.

43. Acha A, Ruesga MT, Rodriguez MJ, Martínez MA, Aguirre JM, "Aplicaciones de la citología oral por raspado (exfoliativa) en el cáncer y precáncer oral”. Medicina y Patologia Oral 2005;10:95-102.

44. Diniz M, Garcia Garcia A, Crespo A, Martins JL, Gándara JM, "Aplicaciones de la citología exfoliativa en el diagnóstico de cáncer oral". Med Oral 2004;9:355-61.

45. Konstantin S, Follen M, Aaron J, Pavlova I, Malpica A, Lotan R, Richards-Kortum, R: "Real-Time Vital Optical Imaging of Precancer Using Anti-Epidermal Growth Factor Receptor Antibodies Conjugated to Gold Nanoparticles". Cancer Research 2003;63:1999-2004.

46. Shannon E, Weigum P, Floriano N, Christodoulides N, McDevitt JT: "S based sensor for analysis of EGFR biomarker expression in oral cancer". Lab Chip 2007; 7:995-1003. 